\title{
Complete topological analysis of the periodic structures in a harmonically driven bubble oscillator near Blake's critical threshold: infinite sequence of two-sided Farey ordering trees
}

\author{
Ferenc Hegedüs ${ }^{\mathrm{a}, *}$ \\ ${ }^{a}$ Budapest University of Technology and Economics, Faculty of Mechanical Engineering, \\ Department of Hydrodynamic Systems, P.O. Box 91, 1521 Budapest, Hungary
}

\begin{abstract}
The topology of the stable periodic orbits of a harmonically driven bubble oscillator, the Rayleigh-Plesset equation, in the space of the excitation parameters (pressure amplitude and frequency) has been revealed numerically. This topology is governed by a hierarchy of two-sided Farey trees initiated from a unique primary structure defined also by a simple asymmetric Farey tree. The sub-topology of each of these building blocks is driven by a homoclinic tangency of a periodic saddle. This self-similar organization is a suitable basis for a general description, since it is in good agreement with partial results obtained in other periodically forced oscillators and iterated maps. The applied ambient pressure in the model is near but still below Blake's critical threshold. Therefore, this paper is also a straightforward continuation of the work of Hegedüs [1], who first found numerical evidence for the existence of stable, period 1 solutions beyond Blake's threshold. The present findings are crucial for the extension of the available numerical results from period 1 to arbitrary periodicity.
\end{abstract}

Keywords: bubble dynamics, bifurcation structure, nonlinear dynamics, parameter continuation, topology, Farey ordering

\footnotetext{
*Corresponding author. Tel: +36 1463 1680; fax: +36 14633091

Email address: hegedusf@hds.bme.hu (Ferenc Hegedüs)
} 


\section{Introduction}

Pattern recognition. This is one of the most powerful skills of mankind having a great contribution to reach our current technological and scientific level. For instance, without the recognition of stellar constellations and understanding of weather cycles, the rise of civilized empires would not have been possible. More specifically, pattern recognition is important, for instance, in the statistical analysis of financial time series to identify the dynamics of stock market crashes [2], in the prediction of earthquakes by determining patterns of time series (such as fractal behaviour) in stick-slip models [3].

Thus, pattern recognition plays a role in many branches of science, and nonlinear dynamics is not an exception. The extensive study of periodically driven nonlinear oscillators in the last few decades have revealed several unique features of these systems. As computer capacities increased, more and more information has been accumulated about the bifurcation structures of various systems, and summarised in many textbooks [4-6]. Some of these classical models are the Duffing, Van der Pol or Toda oscillators. The huge number of numerical investigations have uncovered several topological universalities in the bifurcation structure with respect to a single control parameter. For instance, the standard Feigenbaum period-doubling cascade [7], the alteration of regular periodic and chaotic motions through different kinds of crises [8], or the topological templates of chaotic motions [9].

The real challenge now is to find universalities in a two-dimensional parameter space. In the early stage of investigations, the endoskeletons of the domains of some simple periodic oscillations were computed by using the efficient continuation method. Although they could not reveal the fine bifurcation structures, the appearance of resonance horns organized as a Farey ordering tree for small amplitude oscillations [10] or the universal description of the formation of stable periodic orbits above the first subharmonic resonance [11] in the amplitude-frequency parameter plane provided valuable information about the system behaviour.

The exponentially increasing computational resources allowed computing high resolution bi-parametric plots with the shooting method; see for example the pioneering work of Bonatto et al. [12] and Lauterborn et al. [13]. Here, at every parameter pair a simulation was started by using an initial value problem solver with suitable initial conditions, and after con- 
vergence to a stable solution some properties are recorded. For instance, positive values of the Lyapunov exponent indicate the regions with chaotic solutions. These very resource-intensive computations showed specific features of bi-parametric plots, such as the existence of isoperiodic stable structures immersed in large chaotic domains. These structures are usually form shrimp-shaped domains or denominated Arnold tongues, and they can be organized in several ways, such as Fibonacci-type series and Golden ratio for Arnold tongues [14], located along a specific direction with an accumulation point [15], or they can form zig-zag pattern [16]. In spite of the already existing huge amount of numerical results, no general rule has been found for the organization of these structures. They can differ from system to system, and year by year new patterns are discovered. This suggests that we are far from global understanding of bi-parametric structures, not to mention higher dimensional parameter spaces.

The present study intends to add a piece to the puzzle of the global picture of two-parametric bifurcation structures in the excitation amplitudefrequency parameter plane of a harmonically excited system, namely, the Rayleigh-Plesset equation which is a spherical bubble oscillator. Via thorough numerical investigations by combining the shooting and the pseudoarclength continuation techniques (for more details see Chapter 10 of [17]), it has been revealed that the topological description of the bifurcation pattern of stable periodic oscillations can be determined provided that excitation frequency is above the first subharmonic resonance frequency of the system. This structure is defined by an infinite sequence of two-sided Farey ordering trees [18] and Feigenbaum cascades [7] based on a primary structure, which is an asymmetric Farey tree and serves as an endoskeleton of the whole bifurcation pattern. The topology of each two-sided Farey tree is governed by the homoclinic tangency of the invariant manifolds of a saddle-type periodic orbit $[17,19]$. These findings are in good agreement with the results of other models, such as, complex maps [20], Duffing [21] or Toda [11] oscillators. Still, this is the first study to put the partial results together and to give a more general explanation of the bifurcation structure and the validity range in the two-dimensional parameter space.

The present investigation is also a natural continuation of Hegedüs [1], who found stable periodic solutions beyond Blake's critical threshold without applying any linearization or other reduced order modelling. The next subsection intends to summarise this topic briefly. 


\subsection{Blake's critical threshold}

The equilibrium radius of a gas-filled bubble is governed by the mechanical balance

$$
0=p_{V}-P_{\infty}+p_{g o}\left(\frac{R_{o}}{R_{E}}\right)^{3 n}-\frac{2 \sigma}{R_{E}}
$$

at the bubble interface, where $R_{E}$ is the equilibrium bubble radius. The vapour and the ambient pressures are $p_{V}$ and $P_{\infty}$, respectively. The surface tension is $\sigma$. The polytropic exponent $n$, the gas reference pressure $p_{g o}$ and radius $R_{o}$ determine the mass of gas $m_{G}$ within the bubble.

The equilibrium radius with respect to the tension $p_{V}-P_{\infty}$ at ambient temperature $T_{\infty}=37^{\circ} \mathrm{C}$ is presented in Fig. 1 . The curve contains a turning point called Blake's critical threshold, first identified by Blake [22], which separates the stable $R_{E}^{s}$ (solid line) and unstable $R_{E}^{u}$ (dashed line) branches. Beyond this threshold the bubble tends to grow indefinitely regardless of its initial state as a consequence of the absence of both the stable and the unstable equilibrium radii.

The experimental work with cryogenic liquids of Marston and Greene [23], however, implied that such bubbles can be stabilized by applying a harmonically varying pressure field on the liquid domain (acoustic irradiation). For decades, many authors attempted to find numerical evidence for this stabilisation mechanism only with partial success. Gumerov [24], for instance, found stable oscillations beyond Blake's threshold but the applied third order approximation limited the findings only to relatively small amplitude oscillations. Although Hao and Prosperetti [25] used no reduced order modelling, the applied numerical technique was practically unsuitable to find any stable solutions even after thousands of simulated cycles. The first break-through was achieved by Hegedüs [1], who was able to stabilize the bubble without linearization or other reduced order modelling by using the well-known Rayleigh-Plesset equation.

Although the results of Hegedüs [1] are important for the understanding of the stabilization mechanism, they are restricted to a special kind of solution called period 1 oscillation (the period is equal to the period of the excitation). The seeking process was rather simple. Below the critical threshold, stable period 1 oscillations emerge from the stable equilibrium radius as the excitation pressure amplitude $p_{A}$ starts to increase from zero. With the pseudo-arclength continuation technique, the complete exploration of the stable period 1 domains in the excitation pressure amplitude-excitation 
frequency $\left(p_{A}-\omega\right)$ parameter plane could be determined. By gradually decreasing the ambient pressure beyond the critical threshold, it was observed that only one large stable period 1 domain remained.

The present findings are very important from this stabilisation point of view, since a large stable period 1 domain beyond Blake's threshold was found at the higher frequency regions. This implies that results can be extended to arbitrary periodicities described topologically in more detail in the next sections.

It is worth emphasizing that the system under such conditions (low pressure) is not-strictly dissipative, implying that stable bubble motions are not guaranteed, see also the review of Feng and Leal [26]. Such bubble behaviour (near Blake's threshold) has already been investigated by Chang and Chen [27] and Smereka et al. [28] with Hamiltonian formalism near the resonance frequency but a detailed topological analysis in the pressure amplitude-frequency $\left(p_{A^{-}}-\omega\right)$ parameter space is still missing in the literature.

\section{Formulation of the problem}

The applied bubble model in this paper is the well-known RayleighPlesset equation [29] written in the form

$$
R \ddot{R}+\frac{3}{2} \dot{R}^{2}=\frac{1}{\rho_{L}}\left(p_{L}-p_{\infty}(t)\right),
$$

where $R(t)$ is the time dependent bubble radius, $\rho_{L}$ is the liquid density, $p_{L}$ and $p_{\infty}(t)$ is the pressure at the bubble wall and far away from the bubble, respectively. The dot stands for the derivation with respect to time $t$. The time-dependent far field pressure

$$
p_{\infty}(t)=P_{\infty}+p_{A} \sin (\omega t)
$$

is a sum of the static ambient pressure $P_{\infty}$ and the harmonic forcing with pressure amplitude $p_{A}$ and angular frequency $\omega$. The bubble interior is a mixture of vapour and non-condensable gas. Both are treated as ideal gases, therefore, the pressure inside the bubble is the sum of their partial pressures:

$$
p_{G}+p_{V}=p_{L}+\frac{2 \sigma}{R}+4 \mu_{L} \frac{\dot{R}}{R},
$$

where $\sigma$ is the surface tension and $\mu_{L}$ is the liquid dynamic viscosity. The vapour pressure depends on the ambient temperature $T_{\infty}$ which was constant 
during the computations. The gas content inside the bubble follows a simple polytropic relationship

$$
p_{G}=p_{g o}\left(\frac{R_{o}}{R}\right)^{3 n}
$$

with a polytropic exponent $n=1.4$ (adiabatic behaviour). The reference properties $p_{g o}$ and $R_{o}$ determine the mass of gas $m_{G}$ inside the bubble, that is, the size of the bubble. The material properties of the liquid water $\left(\rho_{L}, p_{V}\right.$, $\left.\sigma, \mu_{L}\right)$ were calculated by means of the Haar-Galagher-Kell equation of state [30], at an ambient temperature $T_{\infty}=37^{\circ} \mathrm{C}$ and pressure $P_{\infty}=5458 \mathrm{~Pa}$. The choice of the ambient pressure is explained in the next subsection.

\subsection{Equilibrium radius, parameters and the dimensionless system}

The equilibrium bubble radius $R_{E}$ of the unexcited system $\left(p_{A}=0\right)$ as a function of the tension $p_{V}-P_{\infty}$ is shown in Fig. 1, and computed by means of Equation (1), for a given mass of gas $m_{G}$ and ambient temperature $T_{\infty}=37^{\circ} \mathrm{C}$. The curve contains a turning point, called Blake's critical threshold marked by the black dot, which separates the stable $R_{E}^{s}$ (solid) and unstable $R_{E}^{u}$ (dashed) branches at the critical radius $R_{C}$. In the paper of Hegedüs [1] it has been derived that the critical radius, which is chosen to be $R_{C}=0.1 \mathrm{~mm}$, determines the gas reference pressure

$$
p_{g o}=\frac{2 \sigma}{3 n R_{C}}
$$

the mass of the gas content

$$
m_{G}=\frac{8 \sigma R_{C}^{2} \pi}{9 n \Re T_{\infty}}
$$

( $\Re$ is the specific gas constant) and thus the size of the bubble, and the critical tension

$$
p_{V}-P_{C}=\frac{2 \sigma}{R_{C}} \frac{3 n-1}{3 n}
$$

provided that the arbitrarily specifiable gas reference radius is $R_{o}=R_{C}$.

By defining a dimensionless relative pressure as

$$
P_{R}=\frac{p_{V}-P_{\infty}}{p_{V}-P_{C}}
$$




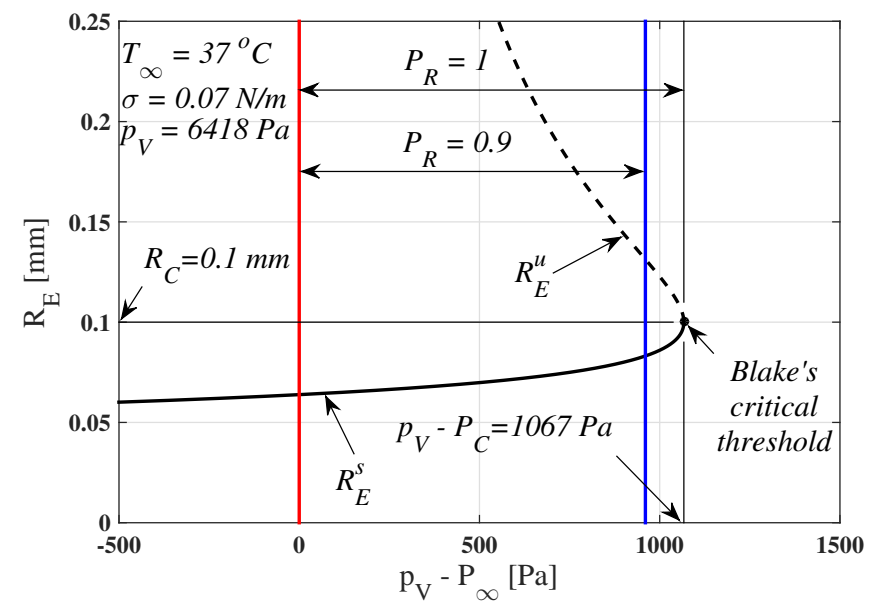

Figure 1: Typical equilibrium bubble radius curves for water at $T_{\infty}=37^{\circ} \mathrm{C}$ as a function of tension $p_{V}-P_{\infty}$. The solid and dashed lines are the stable and unstable equilibrium radius curves, respectively. The black dot denotes Blake's critical threshold.

the ambient pressure $P_{\infty} \in\left(p_{V}, P_{C}\right)$ can be transformed into $P_{R} \in(0,1)$, see also Fig. 1. The region $P_{R}>1$ does not have any equilibrium radius, neither stable nor unstable, leading to steadily growing bubbles to infinity. This region has been the subject of keen interest because of the issue of bubble stabilisation with harmonic forcing discussed in the Introduction. From the stabilisation mechanism point of view, however, the domain $0<P_{R}<1$ is much more important. At $P_{R}=0.9$, Hegedüs [1] successfully explored all the period 1 solutions, bifurcated from the equilibrium radii $R_{E}^{s}$ and $R_{E}^{u}$ as $p_{A}$ started to increase from zero, in the pressure amplitude $p_{A}$ - frequency $\omega$ plane. Then, by gradually decreasing $P_{\infty}$ beyond Blake's threshold the stable domains which survive the procedure could be easily determined with the effective pseudo-arclength continuation technique. This paper focuses on the topological description of the bifurcation structure of the stable periodic solutions at $P_{R}=0.9$, which is crucial for the extension of the numerical results beyond Blake's threshold to arbitrary periodicity. At the applied relative pressure, the tension is $p_{V}-P_{\infty}=1067 \mathrm{~Pa}$, as shown by the blue vertical line in Fig. 1, resulting in $P_{\infty}=5458 \mathrm{~Pa}$.

Introducing dimensionless time $\tau$, dimensionless bubble radius $y_{1}$ and 
dimensionless bubble wall velocity $y_{2}$ defined as

$$
\begin{aligned}
\tau & =\frac{t}{\frac{2 \pi}{\omega}}, \\
y_{1} & =\frac{R}{R_{C}}, \\
y_{2} & =\frac{\dot{R}}{\frac{R_{C} \omega}{2 \pi}},
\end{aligned}
$$

the governing second order nonlinear differential equation (2) can be rewritten as a system of first order dimensionless equations:

$$
\begin{aligned}
& y_{1}^{\prime}=y_{2}, \\
& y_{2}^{\prime}=\frac{p_{L}-p_{\infty}(t)}{p_{\text {ref }}} \frac{1}{y_{1}}-\frac{3}{2} \frac{y_{2}^{2}}{y_{1}},
\end{aligned}
$$

177 where' stands for the derivative with respect to the dimensionless time $\tau$. 178 The reference pressure is

$$
p_{\text {ref }}=\rho R_{C}^{2}\left(\frac{\omega}{2 \pi}\right)^{2} .
$$

The polytropic relationship of the gas content is

$$
p_{G}=\frac{2 \sigma}{3 n R_{C}}\left(\frac{1}{y_{1}}\right)^{3 n} .
$$

181

Finally, the harmonic excitation far away from the bubble is

$$
p_{\infty}(t)=P_{\infty}+p_{A} \sin (2 \pi \tau) .
$$

182 Note that in the dimensionless system, the period of the excitation becomes 183 unity $\left(\tau_{o}=1\right)$ according to equation (18). 


\section{Numerical results}

\subsection{Global scan of stable periodic attractors}

The simplest way to obtain a global picture about the stable solutions (attractors) in a parameter range is to take an initial value problem solver and perform numerical simulations at each parameter value with suitably chosen initial conditions. After the convergence of the transient trajectory, the points of the Poincaré sections, obtained by sampling the continuous solution at every integer multiple of the period of the excitation $\tau_{o}$, are recorded for some additional cycles. With the method of Poincare section, the threedimensional continuous dynamics can be transformed into a two-dimensional map. The Poincaré points of the continuous solutions $y_{1}(t)$ and $y_{2}(t)$ are denoted by $\Pi\left(y_{1}\right)$ and $\Pi\left(y_{2}\right)$, respectively.

The employed initial value problem solver was a fourth order Runge-Kutta scheme with fifth order embedded error estimation built in MATLAB (ode45). The absolute and relative error tolerances were both $10^{-9}$. The solutions were relatively smooth in time, see e.g. Fig. 2A, therefore, such non-stiff ordinary differential equation solver was quite suitable. Parenthetically, far away from Blake's critical threshold the bubble oscillators are usually stiff problems due to the large amplitude collapse-like oscillations. In such cases, they must be solved with an implicit scheme. Due to the relatively low dissipation (viscosity) the transients died out approximately within 700 to 1000 acoustic cycles.

During the computations, three kinds of solutions occurred. The most common stable solution is a simple periodic attractor whose periodicity is an integer multiple of the period of the excitation. The dimensionless bubble radius vs. time curves of a period 1 (black) and a period 3 (blue) co-existing attractors are presented in Fig. 2A, where the dots are the sampled Poincaré points. The trajectories in the phase space $\left(y_{1}-y_{2}\right.$ plane) form closed curves, and they can intersect themselves and each other due to the non-autonomous nature of the system, see Fig. 2B. Observe that the number of the Poincaré points in the state space of the period 1 and the period 3 orbits are 1 and 3 , respectively. In the rest of the paper, only the points of the Poincaré map will be presented to avoid overcrowded figures.

Because of the non-strictly dissipative property of the bubble model, there are special, unbounded trajectories where the bubble radius exhibits infinite growth. An example is presented by the red curve and dots in Fig. 2A-B. 

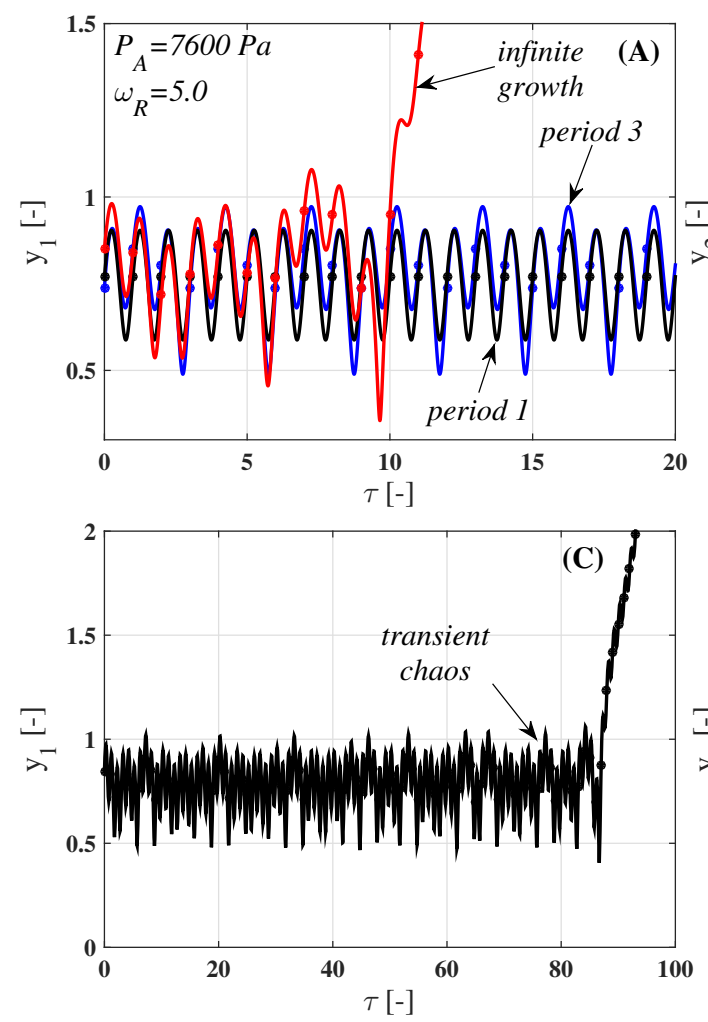
is necessary.
After only 10 cycles, the bubble escapes from the global basin of attraction of the system, its radius tends to increase very rapidly and keeps growing with time. This behaviour makes the hunting for stable oscillations difficult, and thus, the application of several initial conditions at given parameter values
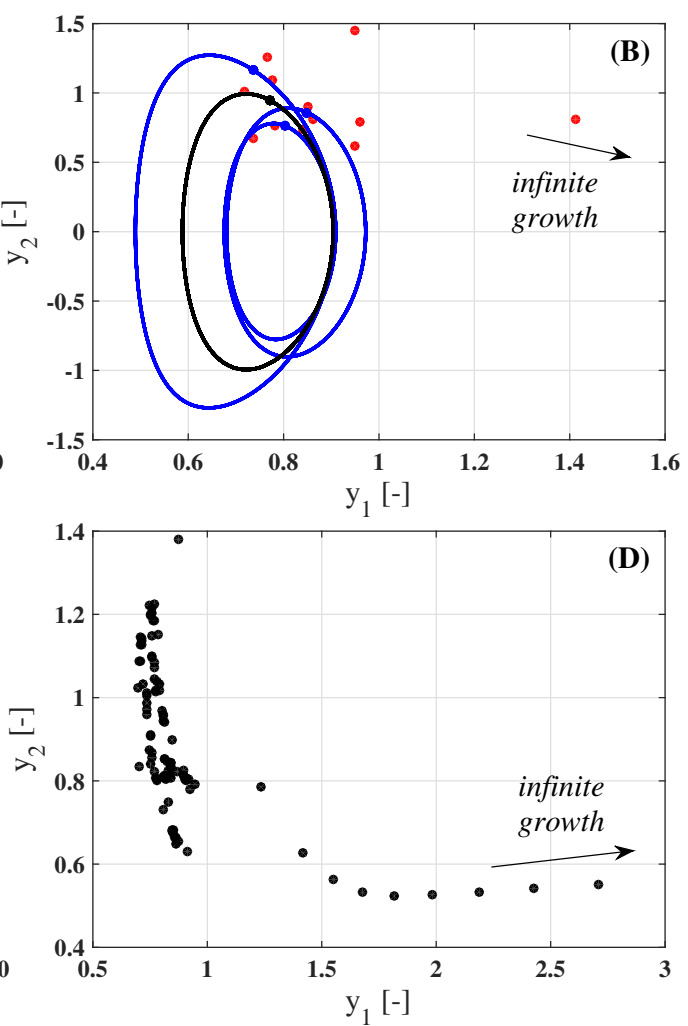

Figure 2: Different kind of solutions of applied bubble model. The upper panels show a period 1, a period 3 and an unbounded co-existing solutions. The lower panels represent an unbounded trajectory initiated near an unstable chaotic solution (transient chaos).

The especially long, seemingly stable transient oscillations prior to the infinite growth of an unbounded trajectory imply the presence of transient (unstable) chaos, see Fig. 2C-D. Such solutions usually cannot be found even if one integrates the system backward in time as they are often chaotic saddles. The determination of their properties, such as the fractal dimension or Lyapunov exponent, needs large computation capacities. The methods based on measuring the escape rate, for instance, require the solution of millions of transient trajectories initiated systematically from a grid of points 
defined on a rectangular domain in the state space. A thorough discussion on the properties of unstable chaos and its effect on the global dynamics of a system can be found in the book of Lai and Tél [31]. These theories can be successfully applied on very complex geometries as well [32]. Although the investigation of transient chaos is beyond the scope of the present paper, its presence makes the monitoring of the convergence difficult. Therefore, the maximum number of allowed acoustic cycles were as high as 1500 which was sufficient for most of the trajectories to escape from the neighbourhood of the unstable chaotic solutions, if there were any.

The bifurcation diagram is a very efficient tool for the analysis of the qualitative change of the different attractors with respect to a parameter. As a global scan of the bifurcations structure, Fig. 3 presents the first component of the Poincaré points $P\left(y_{1}\right)$ at different relative frequencies $\omega_{R}$ with the pressure amplitude $p_{A}$ as control parameter varied between $0 \mathrm{~Pa}$ and $12000 \mathrm{~Pa}$ with an increment of $1 \mathrm{~Pa}$. At every parameter value, 40 randomly chosen initial conditions were used to reveal the co-existing stable solutions. Observe that the achieved maximum pressure amplitudes never reach the prescribed upper limit because of the non-strictly dissipative nature of the system. That means that, in spite of the applied large number of initial conditions, at sufficiently high pressure amplitudes, only unbounded trajectories can be found. Throughout the subfigures, the periodicities of the relevant attractors are denoted by arabic numbers.

The bifurcation structure at relative frequency $\omega_{R}=0.4$ is rather simple. A period 1 attractor emerges from the stable equilibrium radius $R_{E}^{s}$, and it goes through a pair of saddle-node (SN) bifurcations forming a hysteresis at approximately $p_{A}=35 \mathrm{~Pa}$. It becomes unstable at $p_{A}=100 \mathrm{~Pa}$ via a period doubling (PD) bifurcation, and the evolved period 2 attractor exists only up to $p_{A}=110 \mathrm{~Pa}$ pressure amplitude. In a very narrow parameter range, a period 3 stable solution co-exists with the dominant period 1 orbit. Increasing the relative frequency to $\omega_{R}=1.0$, the hysteresis of the period 1 curve disappears, and a period 2 and a period 3 domain arise in the region of small pressure amplitude.

At higher relative frequencies, above $\omega_{R}=2.0$ (Fig. 3C-F), the attractors survive larger pressure amplitudes. At $\omega_{R}=5.0$, stable domains exist up to $p_{A}=10000 \mathrm{~Pa}$. In parallel, the period doubling bifurcation of the period 1 solution is shifted towards larger pressure amplitudes, that is, the parameter region of the existence the fundamental period 1 orbit increases with the relative frequency. Within this domain, regular patterns in the bifurcation 

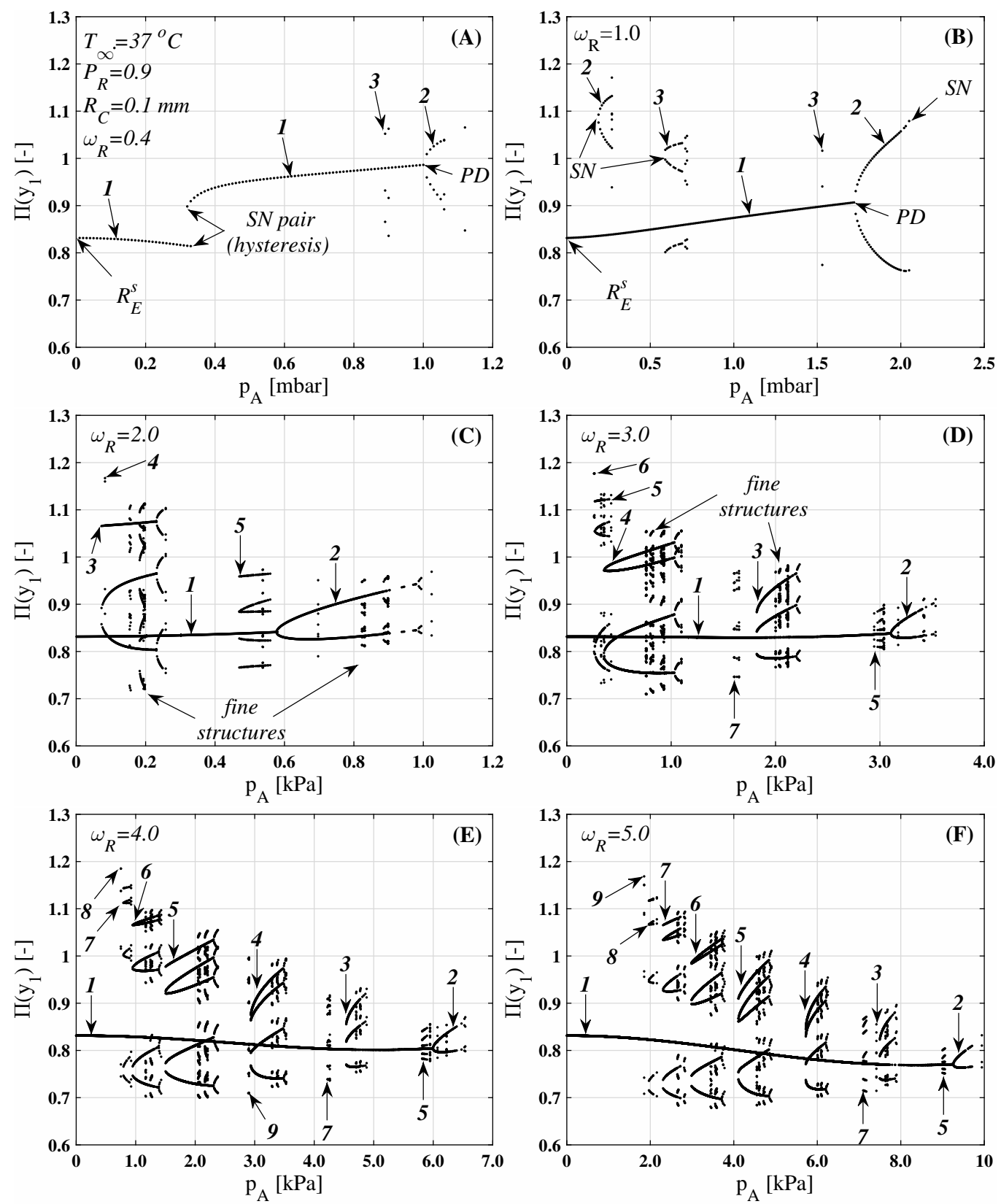

Figure 3: The first component of the Poincaré section $P\left(y_{1}\right)$ of the attractors at different relative frequencies $\omega_{R}$ with respect to the pressure amplitude $p_{A}$ as control parameter. The numbers denote the periodicity of the attractors. 
diagram of periodic attractors with a large variety of periodicities can be detected whose complex structure continuously unfolds as the relative frequency increases. In the following, the rules of organisation of these periodic orbits at such high frequencies will be systematically explored, and the full topological description of the bifurcation pattern will be given.

The simple initial value problem solver, applied here, has been used for decades to investigate the bifurcation structure of various bubble oscillators with a variety of control parameters. The interested reader is referred to the publications [1, 33-46].

\subsection{The main structure}

In Fig. 4, the magnification of Fig. 3F is presented for a better overall view of the bifurcation structure. The lower subfigure shows the period of the found attractors $(N)$ which help identify the periodicities and the bifurcation patterns. The main structure is composed by orbits with relatively low periodicities marked by the arabic numbers in the lower panel of Fig. 4 . They appear via SN bifurcations (except the period 2 solution which originates from the period 1 curve via period-doubling bifurcation) and their period gradually increases one by one towards the lower pressure amplitudes up to period 9. Observe that the SN bifurcations seem to approach to an accumulation point exists somewhere between $1000 \mathrm{~Pa}<p_{A}<2000 \mathrm{~Pa}$ pressure amplitude. We shall see that this plays an important role in the dynamics explained later. Each periodic block in the main structure exhibits an unfinished Feigenbaum period doubling cascade indicating the presence of the aforementioned transient chaos. Moreover, between the SN and the first PD bifurcation in each block, there is a fine structure with very high periodicities (up to even period 50) highlighted in the lower panel of Fig. 4 for the period 5 block.

Besides periodicity, a more powerful tool to analyse the topology is the winding number $w$ which describes the average number of twists of a nearby trajectory around a given solution during one period of the excitation. By straightforward calculation, the winding number can be expressed as fraction of $w=M / N$, where $M$ is the torsion number (the number of twists of a nearby trajectory) and $N$ is the period of the solution, for the details of the computation see e.g. [47]. In general, the winding number is a real positive number, but near saddle-node and period-doubling bifurcations it becomes rational due to the real Floquet multipliers [9], and more importantly it is 

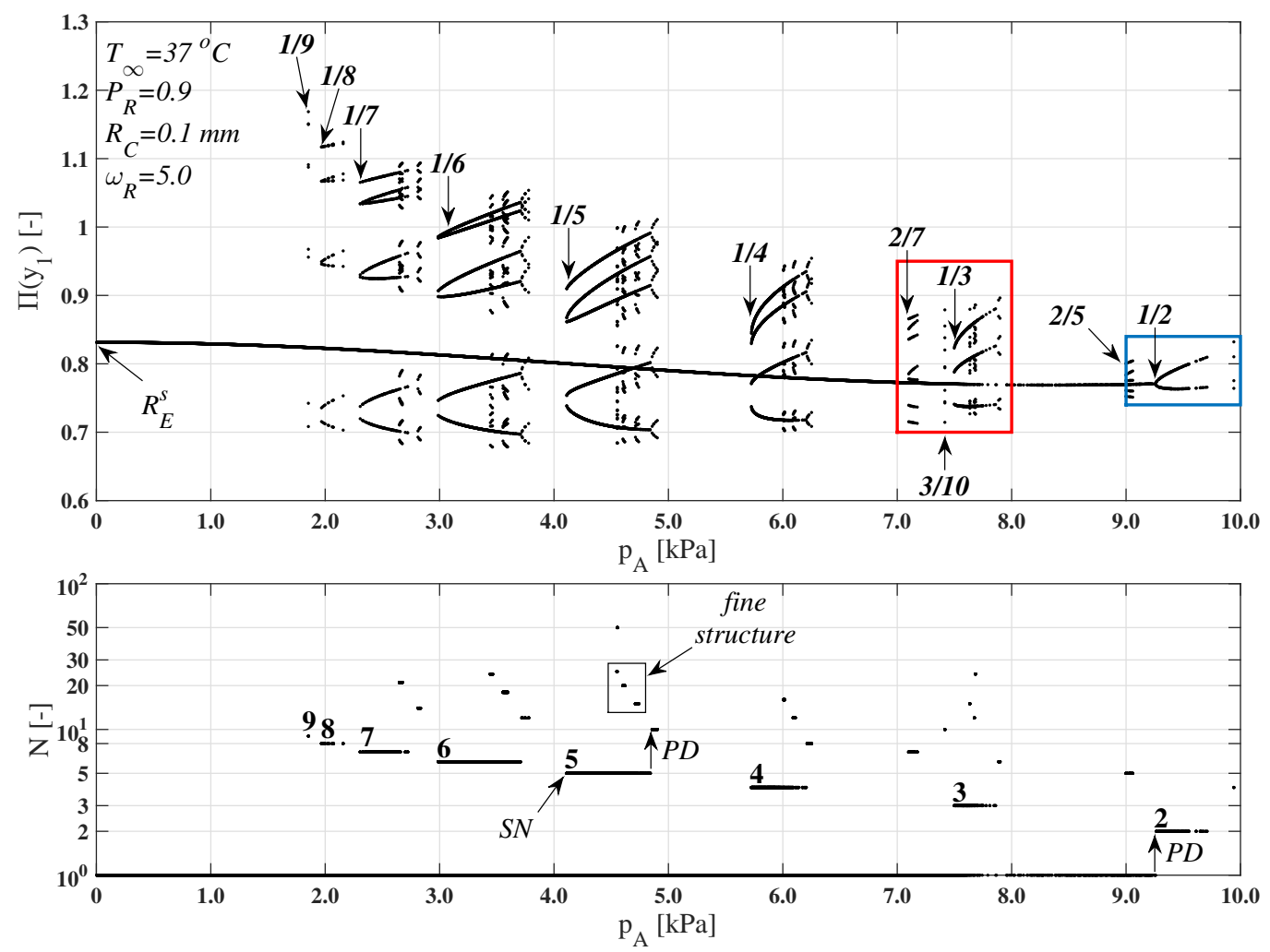

Figure 4: Magnification of the structure of the periodic attractors at $\omega_{R}=5.0$ (upper panel). Periodicity of the attractors (lower panel).

invariant for a wide range of parameters. The winding numbers corresponding to the SN bifurcations of the main blocks and the first PD point of the period 1 solution are denoted by the italic bold fractions in the upper panel of Fig. 4. This invariant quantity was successfully applied to classify the endoskeleton of the bifurcation structures of different oscillators, for instance, Duffing [47-49], van der Pol [50], Toda [51, 52], different bubble oscillators $[34,41,53]$ or even comparison of several nonlinear oscillators [10].

To completely understand the mechanisms that build up the topology of the stable periodic orbits, one needs to compute the unstable periodic solutions as well. An efficient way to do that, is to reformulate the problem into a boundary value problem (BVP) by specifying periodic boundary conditions for the system (13)-(18): 


$$
\begin{aligned}
& y_{1}(0)=y_{1}\left(N \tau_{o}\right), \\
& y_{2}(0)=y_{2}\left(N \tau_{o}\right),
\end{aligned}
$$

where $N$ is the periodicity of the desired orbit. Keep in mind that $\tau_{o}=1$ is the period of the dimensionless excitation, see again equation (18). With a boundary value problem solver, the periodic orbit can be obtained directly, independently of its stability. During the computations, the AUTO bifurcation analysis software was used [54], which discretizes the problem by the method of orthogonal collocation using piecewise polynomials with $2-7$ collocation points per mesh interval [55]. The mesh is automatically refined to the solution according to the local discretization error [56]. In our case, the relative error was given as $10^{-10}$. A thorough investigation on its capabilities were carried out by Hegedüs [1] and Hegedüs and Klapcsik [46]. In the field of bubble dynamics, this powerful software was also used by Fyrillas and Szeri [57] for the rectified diffusion problem, Lauterborn and co-workers to investigate the bifurcation patterns of a single bubble [58], and Hös and co-workers to study the stability of compression systems and pressure relief valves [59-61].

AUTO uses pseudo-arclength continuation to follow solution families (bifurcation curves) with respect to a control parameter. From the stable equilibrium radius $R_{E}^{s}$, for instance, a complete stable period 1 bifurcation curve can be traced as a function of the pressure amplitude $p_{A}$, shown by the lowest black curve in Fig. 5. Observe that here the maximum of the dimensionless bubble radius $y_{1}^{\max }$ is presented in the vertical axis. Since AUTO can handle a periodic solution only as a whole object, the representative picture of the Poincaré points has been lost. As the BVP solver is insensitive to the stability, the unstable part of the period 1 curve can be continuated forward indicated by the small red segment above $p_{A}=9000 \mathrm{~Pa}$. In Fig. 5, the stable and unstable branches are marked by black and red curve sections, respectively. The bifurcation points where the change of stability takes place can be detected as well. In Fig. 5, the saddle-node (SN) and the perioddoubling (PD) bifurcations are denoted by dots and crosses, respectively. With a suitable branch switching algorithm, the family of the period 2 orbits, originated from the PD point of the period 1 curve, was computed up to $p_{A}=10000 \mathrm{~Pa}$ pressure amplitude which also contains a PD point. It is important to emphasize that a saddle-type period 1 branch is evolved from 


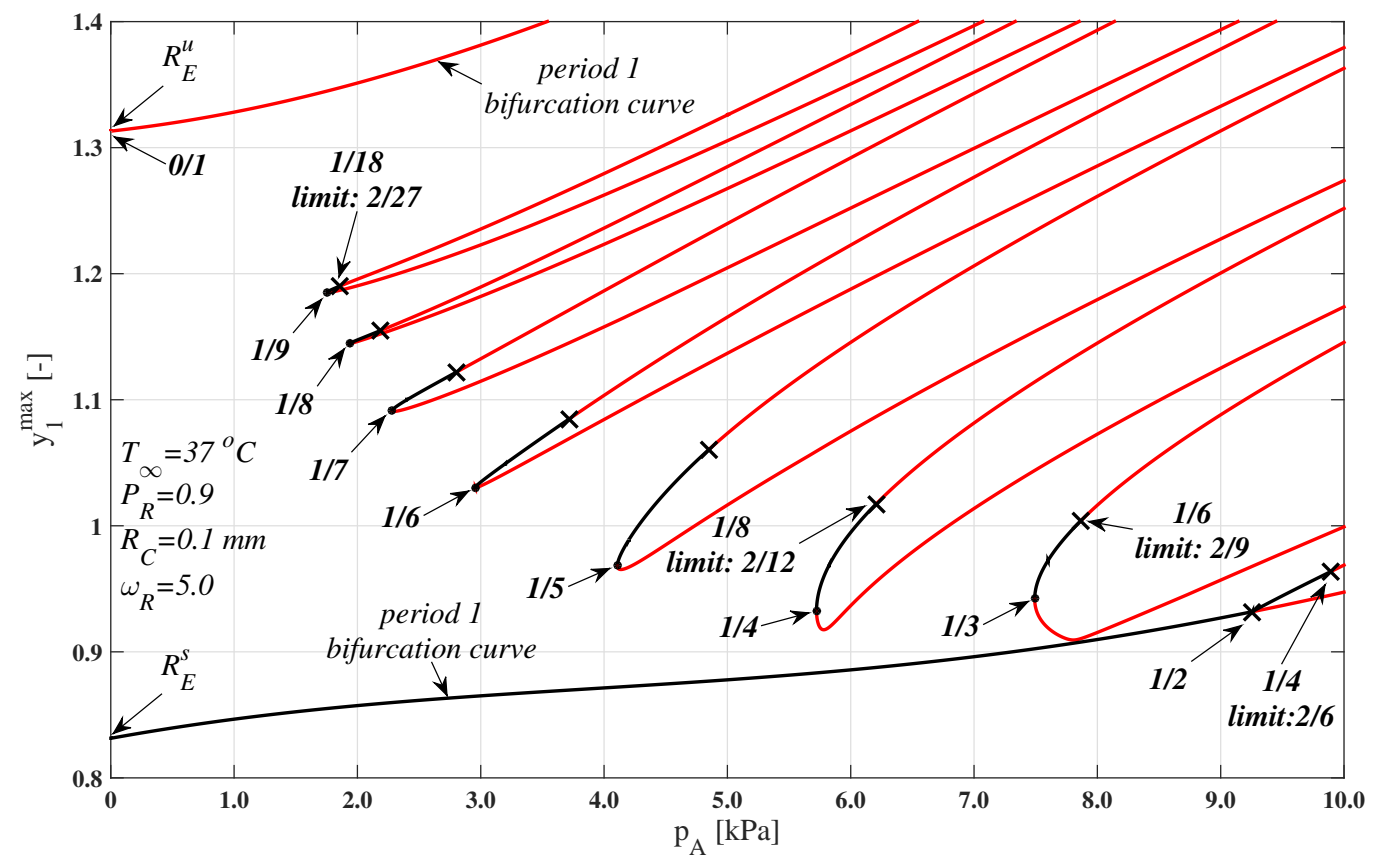

Figure 5: Bifurcation curves of the main structure at $\omega_{R}=5.0$ computed by the boundary value problem solver AUTO. The black and red curves are the stable and unstable solutions, respectively. The saddle-node and period-doubling bifurcations are marked by dots and crosses, respectively.

the unstable equilibrium point $R_{E}^{u}$ presented by the upper red curve in in Fig. 5.

Similarly, all the periodic orbits from period 3 up to period 9 of the main structure were computed and presented in Fig. 5. They are composed of a single branch containing a turning point, which is an SN bifurcation, and a period-doubling point. Between the two bifurcation points, a relatively small segment of stable orbits exist. Keep in mind again that only the maximum values of the whole periodic solutions were recorded. That is, with each solution family only a single curve is associated. The aforementioned winding numbers for the bifurcation points are indicated by the bold fractions. As can be seen, the winding numbers of the main periodic blocks obey a Farey ordering. Between two given bifurcation points characterised by $w_{1}=M_{1} / N_{1}$ and $w_{2}=M_{2} / N_{2}$, another one exists with $w_{3}=\left(M_{1}+M_{2}\right) /\left(N_{1}+N_{2}\right)$. For instance, between the bifurcation points of order $0 / 1$ and $1 / 2$ there is another one with order $1 / 3$. Similarly, between points $0 / 1$ and $1 / 3$ there is 
an SN point with order of $1 / 4$. This topological description is commonly called as Farey ordering tree, summarised in Fig. 6, and it can help identify stable periodic orbits using the pressure amplitude as the control parameter. Therefore, it also seems to be a promising and efficient tool in the hunting for stable solutions beyond Blake's critical threshold. In Fig. 6, the extensions of the period doubling bifurcations of orders $1 / 4$ and $1 / 6$ included only for the better understanding of the forthcoming substructures presented in Fig. 9 and Fig. 11, respectively.

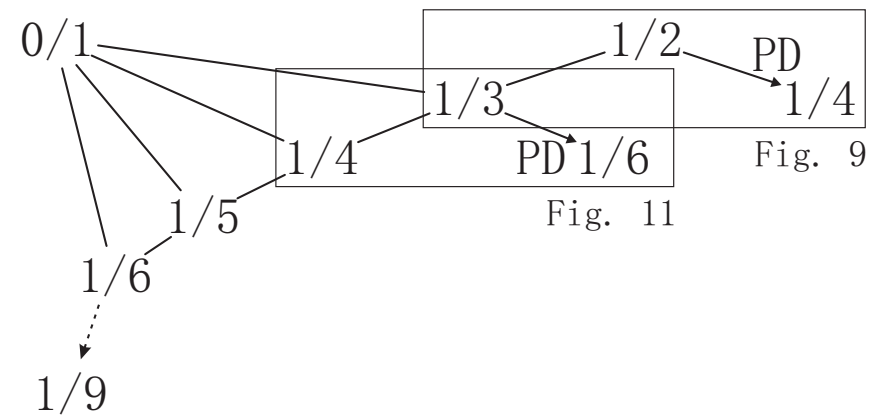

Figure 6: Farey ordering tree of the found periodic attractors of the primary structure. The period doubling bifurcations of order $1 / 4$ and $1 / 6$ serve only for the better understanding of Fig. 9 and Fig. 11, respectively.

It is apparent that the Farey ordering tree describing the topology of the main structure is far from complete. Although there are SN bifurcations with orders of 2/5 and 2/7 appearing also in Fig. 4, which would fit very well into the Farey structure, they were omitted in Fig. 6 with reason. In the next section, it will become clear that such higher order periodic orbits, lying between each adjacent pair of main SN blocks, constitute the well-defined substructures of the system. Therefore, they have a more proper place and role in the overall bifurcation pattern. Such incomplete Farey ordering tree is very common in the literature, for instance, in the case of the forced Duffing oscillator [21]. The structure in the asymmetric Farey tree, presented in Fig. 6 , is governed by the ordinary saddle $R_{E}^{u}$ with order of $0 / 1$, that is, the aforementioned accumulation point of the SN bifurcations, see again Fig. 5, is also governed by the ordinary saddle. Moreover, it is well-known (Wiggins [19]) that near the homoclinic tangency of the stable and unstable manifolds of a saddle-type solution, an infinite number of SN and PD bifurcations accumulate. This accumulation behaviour can be seen both in Fig. 5 (results of AUTO) and in Fig. 6 (Farey ordering tree). Therefore, the organisation of 
and

the stable periodic orbits in the main structure is governed by the homoclinic tangency of the manifolds of the saddle-type periodic orbit bifurcated from the unstable equilibrium point, that is, from the regular saddle $R_{E}^{u}$.

Each stable family of solutions appearing via the SN bifurcation exhibit period-doubling cascades, see the upper panel of Fig. 4. However, after a few PD bifurcations the cascades seemingly terminate without the evolution of any stable chaotic solution. The non-strictly dissipative nature of the system enhances the tendency that solutions with high periodicities have usually smaller domain of attraction. Consequently, they are hard to find with a simple initial value problem solver (shooting method). Although there is no clear evidence for the existence of stable chaotic oscillations evolving via fully developed period-doubling cascades, it is worth discussing the evolutions and the limits of the winding numbers of the PD points in the cascades. There are two winding number routes to the accumulation point of period doubling cascades [47, 62-64], namely,

$$
\begin{aligned}
& w_{k}=w_{\infty}+\frac{(-1)^{k}}{3 m_{0} 2^{k}}, \\
& w_{\infty}=w_{0}-\frac{1}{3 m_{0}},
\end{aligned}
$$

$$
\begin{aligned}
& w_{k}=w_{\infty}-\frac{(-1)^{k}}{3 m_{0} 2^{k}}, \\
& w_{\infty}=w_{0}+\frac{1}{3 m_{0}},
\end{aligned}
$$

where $w_{k}$ is the $k$ th winding number in the cascade, $w_{\infty}$ is the winding number at the accumulation points, $w_{0}$ and $m_{0}$ are the basic winding number and the basic period of the cascade, respectively. The winding numbers of the first PD, and the winding numbers of the accumulation points of some of the PD sequence are presented in Fig. 5. Interestingly, the winding numbers in a PD sequence can also be described by the Farey fractions [65], that is, the Farey sum of two adjacent winding numbers is the winding number at the accumulation point. Therefore, the bifurcation pattern of stable solutions of the main structure can fully be characterised by Farey orderings. 
One is responsible for the development of the $\mathrm{SN}$ bifurcations and the other one governs the PD cascade, and they form a complete endoskeleton of the bifurcation structure of the periodic orbits.

It is worth mentioning that the organization of stable periodic orbits by Farey ordering originates from dynamical systems where the trajectories lie on an invariant torus [53]. The behaviour of such systems is usually governed by the sine circle map, and the Farey ordering is valid provided that the torus exists. Moreover, the presence of Farey structure in the appearance of resonance horns as a function of the excitation frequency $\omega$ is reported by Parlitz et al. [34] and more recently by Hegedús et al. [41].

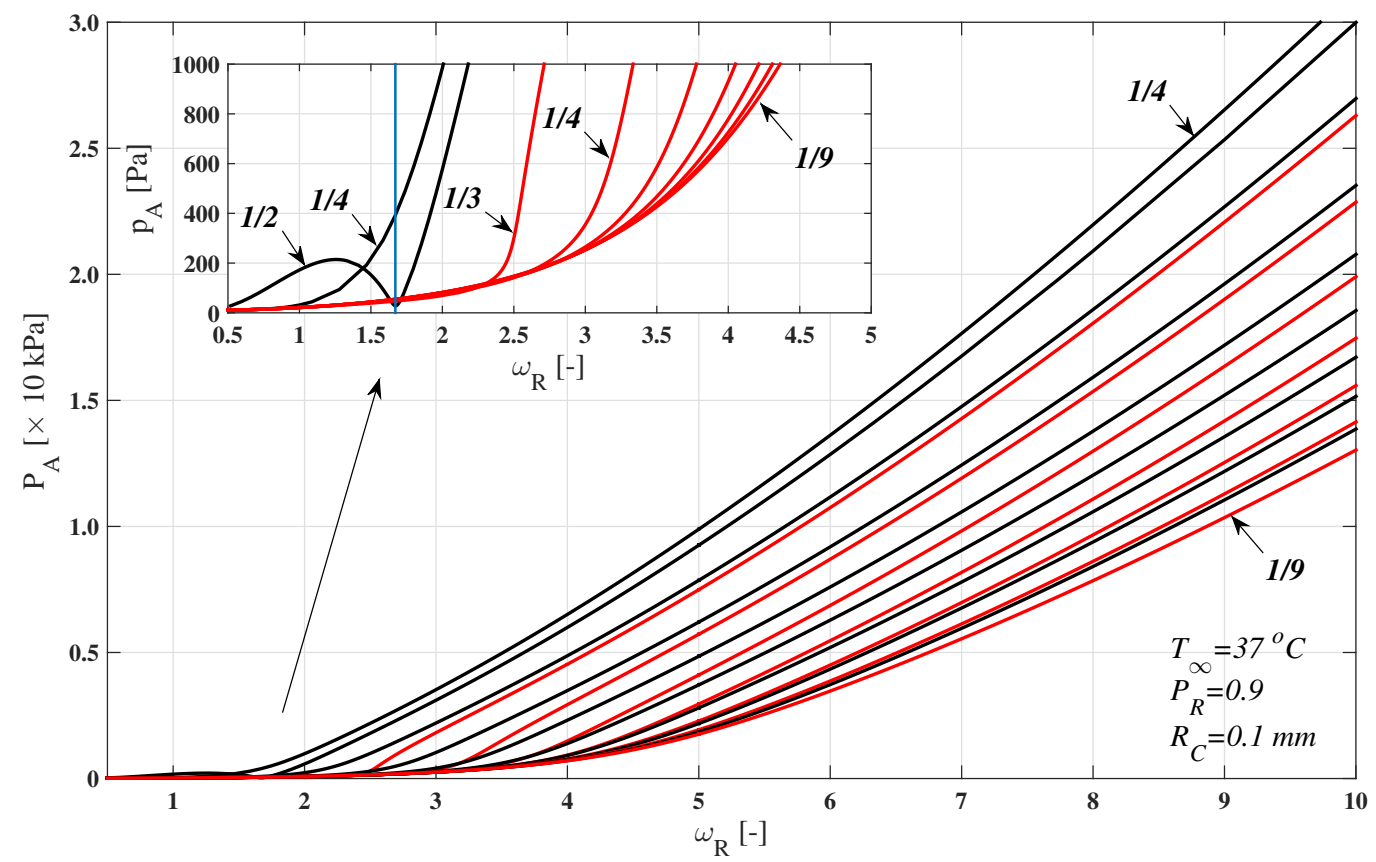

Figure 7: Bi-parametric bifurcation curves of the SN (red curves) and PD (black curves) points found by the BVP solver and presented in Fig. 5.

After the exploration of the topology of the main structure at $\omega_{R}=$ 5.0, the main question is how the found topology changes with the relative frequency $\omega_{R}$ ? In other words, does the topology remain invariant under the change of the parameter $\omega_{R}$ ? The advantage of AUTO is that the detected bifurcation points (SN and PD) can be traced in a two-dimensional parameter space; in our case, in the $\omega_{R}-p_{A}$ plane, by choosing the relative frequency as a secondary control parameter. The results are summarised in Fig. 7, where 
the SN and PD bifurcation points are marked by the red and black curves, respectively. The low frequency, low amplitude region is enlarged in the upper left corner of the figure. In the enlargement, the subharmonic resonant frequency of order $1 / 2$ is represented by the vertical blue line. As usually, the winding numbers are indicated by the bold fractions. The bi-parametric plots in Fig. 7 reveal that the topology remains invariant (the curves do not cross each other) in the high frequency region, approximately above the subharmonic resonance frequency of order $1 / 2\left(\omega_{R}=1.671\right)$. Notice that the higher order subharmonic resonances (e.g. of order $1 / 3$ and 1/4) are far less developed and vigorous than the resonance of order $1 / 2$, and the resonance of order $1 / 9$ is not recognisable at all.

\subsection{Substructures}

The main structure, described in the previous section, defines the endoskeleton of the topology of the stable periodic orbits with respect to the pressure amplitude as control parameter. This topology is invariant under the change of the relative frequency provided that it is higher than the subharmonic resonance frequency of order $1 / 2$. In the following, the topology of the fine substructures, such as those already highlighted in Fig. 3 and Fig. 4, will be clarified. First, let us examine the region near the PD point of order $1 / 2$ between pressure amplitudes $p_{A}=9 \mathrm{kPa}$ and $p_{A}=10 \mathrm{kPa}$. The scan for stable solutions with the IVP solver is summarised in Fig. 8. The increment of the control parameter was reduced from $p_{A}=1 \mathrm{~Pa}$ to $p_{A}=0.1 \mathrm{~Pa}$ in order to obtain a better resolution but the number of initiations per control parameter remained 40. The winding numbers are denoted by the bold fractions, as usual.

The results of Fig. 8 show that near the PD point of order $1 / 2$, the fine structure of the SN bifurcations tend to accumulate to a certain value of the pressure amplitude from both the right and left hand sides. This accumulation point is supposed to be at the previously discussed homoclinic tangency but now corresponding to the saddle-type, period 1 solution evolving through the PD point of order $1 / 2$, see the BVP computations in Fig. 5. Since this family of the solutions is unstable, it does not appear in the IVP scan. Accordingly, the topology of the fine structure can be described by a two-sided Farey tree shown in Fig. 9. This representation of the Farey structure underlines the fact that the topology in this substructure is dominated by the homoclinic tangency of the invariant manifolds of the periodic saddle of order $1 / 2$, and it "collects" the SN bifurcations from both sides. The left hand 


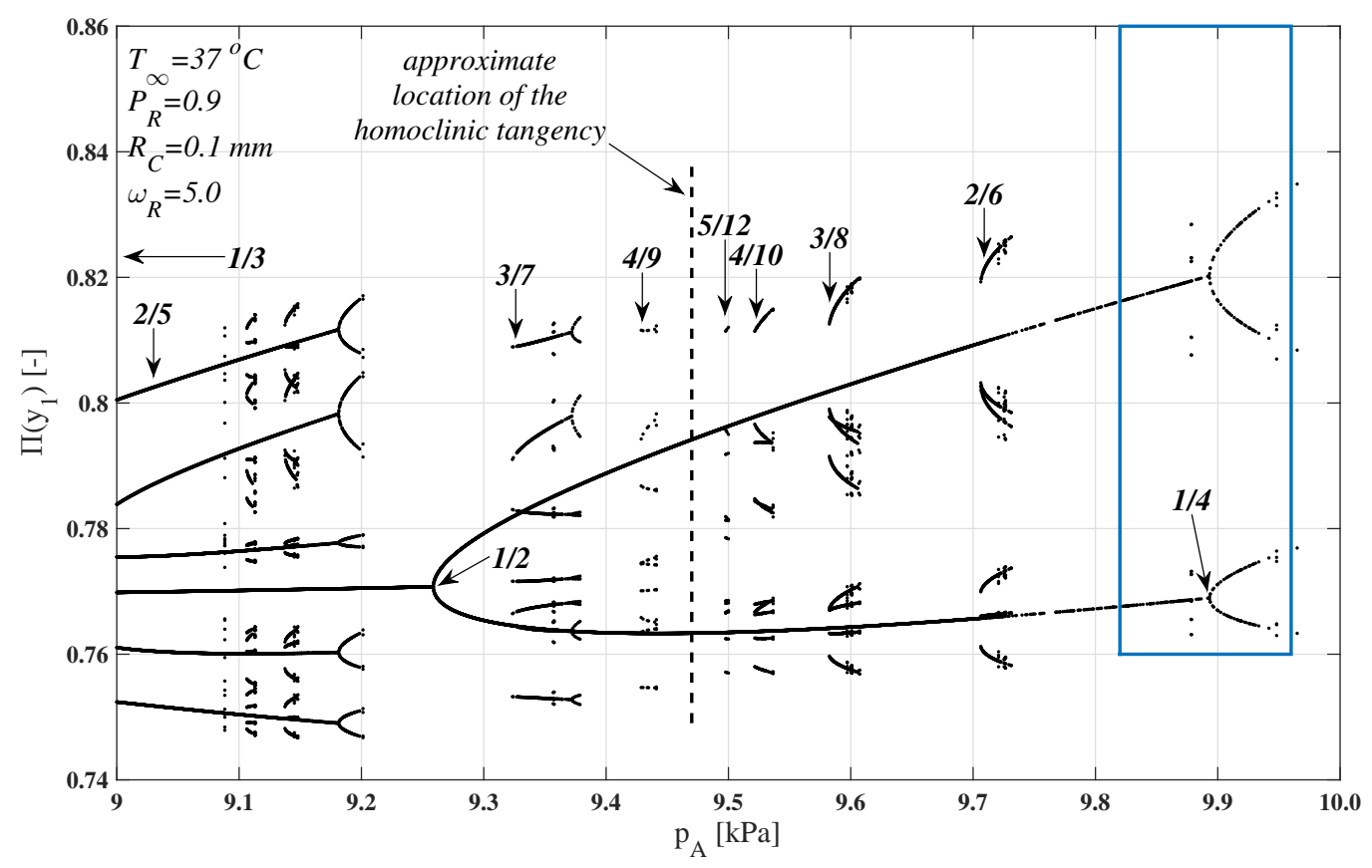

Figure 8: Magnifications of the stable periodic attractors at $\omega_{R}=5.0$, near the PD point of order 1/2 marked also by the first red rectangle in Fig. 4. The increment of the control parameter was $0.1 \mathrm{~Pa}$. The number of initiations in each control parameter was 40 .

Farey structure starts with the SN point of order 1/3 located immediately next to the dominant PD point from left in the main structure, compare Fig. 9 and Fig. 6. In contrast, the right hand side of the Farey structure starts with the next PD point in the Feigenbaum period-doubling sequence, precisely, with the PD point of order 1/4, highlighted also in Fig. 5. The basic elements of this two-sided Farey tree are also highlighted in Fig. 6 by the corresponding rectangle.

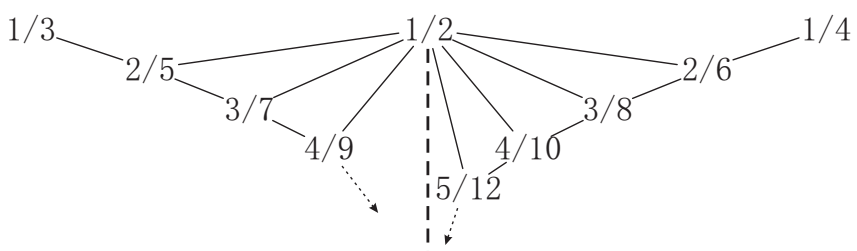

Figure 9: The double-sided Farey ordering tree of the found periodic attractors at $\omega_{R}=5.0$ near the PD point of order $1 / 2$. The structure is dominated by the homoclinic tangency of the invariant manifolds of the periodic saddle of order $1 / 2$. 


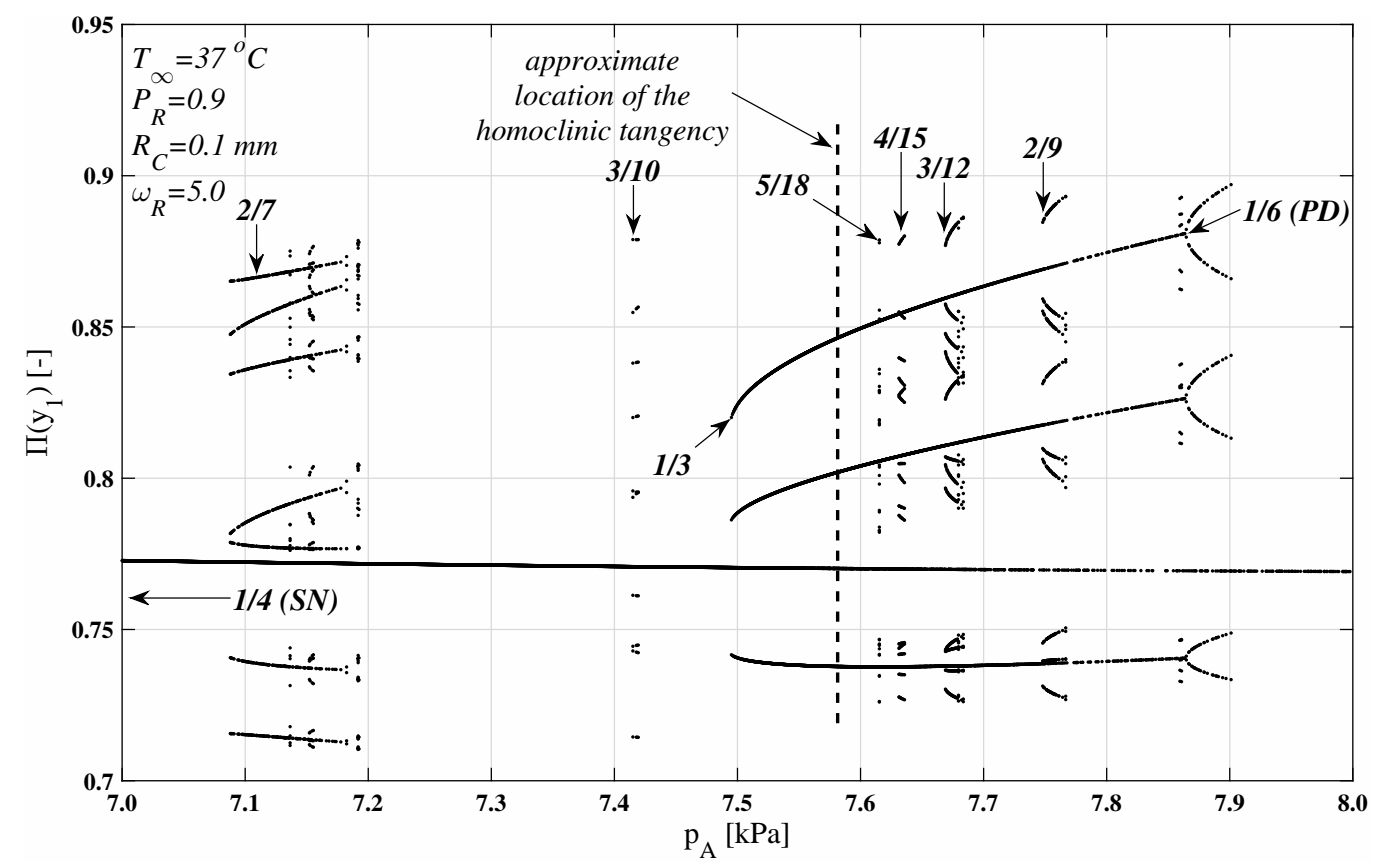

Now, let us examine the topology of the fine structures in a different region of the control parameter $p_{A}$. Figure 10 shows the scan of the stable periodic orbits between pressure amplitudes $p_{A}=7 \mathrm{kPa}$ and $p_{A}=8 \mathrm{kPa}$. The increment of $p_{A}$ was $0.1 \mathrm{kPa}$ but the number of the initial conditions had to increase to 60 for a sufficiently fine resolution of the structures.

Figure 10: Magnifications of the stable periodic attractors at $\omega_{R}=5.0$, near the SN point of order 1/3 marked also by the red rectangle in Fig. 4 . The increment of the control parameter was $0.1 \mathrm{~Pa}$. The number of initiations in each control parameter was 60 .

Similarly to the previous example, an accumulation point of the SN bifurcations can be observed marked by the dashed vertical line in Fig. 10. This accumulation point is at the location of the homoclinic tangency of the invariant manifolds of the period 3 saddle of order $1 / 3$. The unstable saddle again cannot be represented by an IVP scan, it can be shown only in Fig. 5 (BVP calculations) by the red curve originating from the corresponding SN of order $1 / 3$. The topology of the fine structure is described also by a twosided Farey tree. Although the accumulation of the series of SNs in left side is less characteristic than in the previous case, the Farey ordering reveals that the topological description is quite the same. The starting values of the two sides of the Farey tree can also be explained similarly. The left side 
starts with the SN of order $1 / 4$, which is next to the dominating SN point of order $1 / 3$ from left in the main (parent) structure. Meanwhile, the right side again starts with the next PD point in period-doubling sequence. In this case, the basic elements of this two-sided Farey tree are again highlighted in Fig. 6 by the corresponding rectangle.

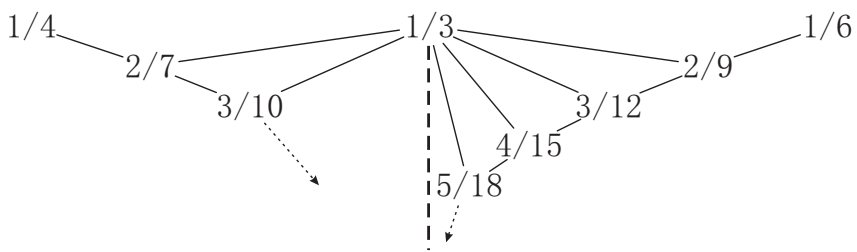

Figure 11: The double-sided Farey ordering tree of the found periodic attractors at $\omega_{R}=$ 5.0 near the PD point of order $1 / 3$. The structure is dominated by the homoclinic tangency of the invarianr manifolds of the periodic saddle of order $1 / 3$.

\subsection{Higher order substructures}

In the previous two subsections, it has been shown that for each member of the main structure (Farey tree) a substructure can be associated described by a two-sided Farey tree. Now, it will be demonstrated that for each element in each substructure there is a sub-substructure which has the same topology as its parent substructure. Figure 12, for instance, represents the usual sequence of stable periodic orbits appeared via SN bifurcations near the PD bifurcation of order $1 / 4$ highlighted by the blue rectangle in Fig. 8. These SN points are governed by the homoclinic tangency of the invariant manifolds of the period 4 saddle originated from the PD point of order $1 / 4$. The increment of the control parameter $p_{A}$ were reduced further to $0.001 \mathrm{~Pa}$. The number of the initial conditions were 60 at each parameter value.

The double-sided Farey tree corresponding to the marked stable periodic orbits in Fig. 12 are presented in Fig. 13. It is clear that it obeys the same rule as in the case of the previously discussed two examples. That is, the left side of the Farey tree starts with the element located next to the dominant saddle from left in the parent substructure, see the SN point of order $2 / 6$ both in Fig. 8 and Fig. 9. And as usual, the right side starts with the next PD point highlighted in Fig. 12. Therefore, it is reasonable to conclude that the subsequent substructures of the unique main structure follow a self-similar topological role, which are nested two-sided asymmetric Farey trees. 


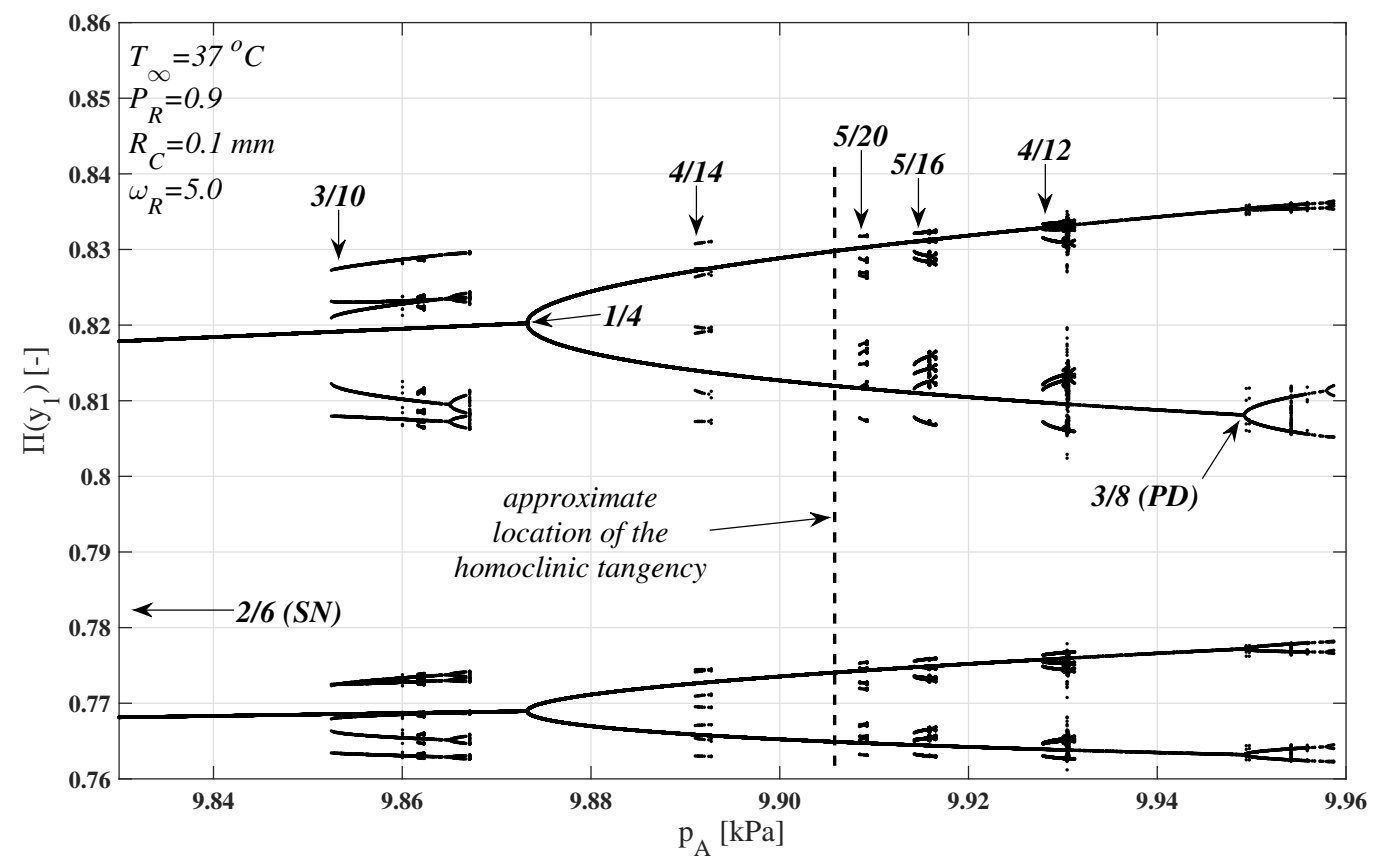

Figure 12: Magnification of the stable periodic attractors at $\omega_{R}=5.0$, near the PD point of order $1 / 4$ marked by the blue rectangle in Fig. 8 . The increment of the control parameter was $0.001 \mathrm{~Pa}$. The number of initiations in each control parameter was 60 .

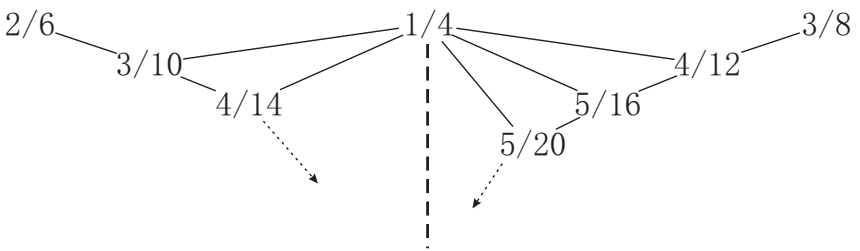

Figure 13: The double-sided Farey ordering tree of the found periodic attractors at $\omega_{R}=$ 5.0 near the PD point of order $1 / 4$ marked in Fig. 12 . The structure is dominated by the homoclinic tangency of the invariant manifolds of the periodic saddle of order $1 / 4$.

\section{Summary}

During the exploration of the dynamics of a harmonically excited gas bubble placed in water at low ambient pressure, the topology of the found periodic attractors was determined in the pressure amplitude-excitation frequency parameter space. It has been found that there is a main or primary structure composed by the subharmonic resonance curves, thus it is valid above the first subharmonic resonance or order $1 / 2$. The organization of the 
stable orbits obeys a simple Farey ordering, which is dominated by the homoclinic tangency of the invariant manifolds of a period 1 saddle bifurcated from the unstable equilibrium point of the unexcited system (the pressure amplitude is set to zero). These stable orbits, appearing via saddle-node bifurcations, exhibit a Feigenbaum period-doubling cascade, which can also be described by a Farey structure. This primary structure is the endoskeleton of the topological description of the stable orbits.

With each element in the main structure (parent), a substructure (child) can be associated composed by a series of saddle-node bifurcations and described by a two-sided Farey tree. The dynamics is again dominated by the homoclinic tangency of the invariant manifolds of a saddle-type, unstable solution corresponding to the element of the parent structure. The left hand side of the Farey tree (child structure) starts with left neighbouring element of the parent structure, while the right hand side starts with the next period doubling bifurcation corresponding to the element in the parent structure. Each element in the substructure then exhibits a period-doubling cascade.

The aforementioned description of the two-sided Farey ordering holds for all the subsequent higher order substructures. That is, for the elements of the substructure and the elements of its own substructure and so forth. The only important thing is to precisely register the associations of the parent and children structures. Summarizing the results, the topology of the stable periodic orbits can be described by a series of repetitive, self-similar Farey ordering trees, provided the frequency is higher than the first subharmonic resonance frequency.

To the best knowledge of the author, the results presented in this paper are the first ones to put the pieces together, obtained by other researchers on different physical models (see the references in the text), and provide a more general topological description of the stable orbits in a wide domain of the bi-parametric space spanned by the excitation frequency and pressure amplitude. That is, the appearance of solutions with high periodicities (in the literature called period- $n$ tupling) are related to the cascade of Farey ordering tree and the domination of the homoclinic tangencies of the invariant manifolds of saddletype unstable orbits. Moreover, the results are a natural extension of the work of Hegedüs [1], who provided evidence for the existence of stable period 1 solutions beyond Blake's critical threshold. Now, there is a possibility to extend those numerical results to arbitrary periodicities and provide a topological description for stable periodic orbits at that parameter region. 
The better understanding of the behaviour of a harmonically excited bubble can support several applications to operate in a more efficient way. These are mainly related to the ultrasonic technology, exploiting the physical phenomenon of bubble generation in a liquid domain by irradiating it with high intensity and frequency ultrasound. Sonochemistry, for instance, is currently regarded as a special trick of modern chemistry to increase the yield of various reaction products even by several hundreds of percentage [66-77]. In food industry, the ultrasonic technology has been successfully applied in pasteurisation [78], to alter the viscosity of many food systems $[79,80]$, and to produce highly stable emulsions by mixing two immiscible liquids $[81,82]$. As a special application, in medicine, the emitted shock waves of ultrasonically excited bubbles can be used to destroy kidney stones (litotropsy) [83], to remove tissues in a targeted area (histotropsy) [84], and to destroy tumour locally in cancer therapy $[85,86]$.

\section{Acknowledgement}

The research described in this paper was supported by the Hungarian Scientific Research Fund - OTKA, Grant No. K81621.

This paper was supported by the János Bolyai Research Scholarship of the Hungarian Academy of Sciences.

\section{References}

[1] F. Hegedüs, Stable bubble oscillations beyond Blake's critical threshold, Ultrasonics 54 (4) (2014) 1113-1121.

[2] D. Sornette, Why Stock Markets Crash: Critical Events in Complex Financial Systems, Princeton University Press, New Jersey, 2004.

[3] E. J. R. Parteli, M. A. F. Gomes, V. P. Brito, Nontrivial temporal scaling in a Galilean stick-slip dynamics, Phys. Rev. E 71 (3) (2005) 036137.

[4] S. H. Strogatz, Nonlinear Dynamics and Chaos with Applications to Physics, Biology, Chemistry, and Engineering, 2nd Edition, Westview Press, Boulder, Colorado, 2014. 
[5] S. Wiggins, Introduction to Applied Nonlinear Dynamical Systems and Chaos, 2nd Edition, Springer-Verlag, New York, 2003.

[6] J. Guckenheimer, P. Holmes, Nonlinear Oscillations, Dynamical Systems, and Bifurcations of Vector Fields, Springer-Verlag, New York, 1983.

[7] M. J. Feigenbaum, Quantitative universality for a class of nonlinear transformations, J. Stat. Phys. 19 (1) (1978) 25-52.

[8] C. Grebogi, E. Ott, J. A. Yorke, Crises, sudden changes in chaotic attractors, and transient chaos, Physica D 7 (1-3) (1983) 181-200.

[9] R. Gilmore, Topological analysis of chaotic dynamical systems, Rev. Mod. Phys. 70 (1998) 1455-1529.

[10] C. Scheffczyk, U. Parlitz, T. Kurz, W. Knop, W. Lauterborn, Comparison of bifurcation structures of driven dissipative nonlinear oscillators, Phys. Rev. A 43 (12) (1991) 6495-6502.

[11] B. K. Goswami, Self-similarity in the bifurcation structure involving period tripling, and a suggested generalization to period n-tupling, Phys. Lett. A 245 (1-2) (1998) 97-109.

[12] C. Bonatto, J. A. C. Gallas, Y. Ueda, Chaotic phase similarities and recurrences in a damped-driven Duffing oscillator, Phys. Rev. E 77 (2) (2008) 026217.

[13] W. Lauterborn, T. Kurz, R. Mettin, P. Koch, D. Krninger, D. Schanz, Acoustic cavitation and bubble dynamics, Arch. Acoust. 33 (4) (2008) 609-617.

[14] S. L. T. de Souza, A. A. Lima, I. L. Caldas, R. O. Medrano-T, Z. O. Guimaräes-Filho, Self-similarities of periodic structures for a discrete model of a two-gene system, Phys. Lett. A 376 (15) (2012) 1290-1294.

[15] A. Celestino, C. Manchein, H. A. Albuquerque, M. W. Beims, Stable structures in parameter space and optimal ratchet transport, Commun. Nonlinear Sci. Numer. Simul. 19 (1) (2014) 139-149. 
[16] R. E. Francke, T. Pöschel, J. A. C. Gallas, Zig-zag networks of selfexcited periodic oscillations in a tunnel diode and a fiber-ring laser, Phys. Rev. E 87 (4) (2013) 042907.

[17] Y. A. Kuznetsov, Elements of Applied Bifurcation Theory, 3rd Edition, Springer-Verlag, New York, 2004.

[18] L. Goldberg, C. Tresser, Rotation orbits and the Farey tree, Ergodic Theory and Dynamical Systems 16 (5) (1996) 1011-1029.

[19] S. Wiggins, Global Bifurcations and Chaos: Analytical Methods, Springer, New York, 1988.

[20] P. Cvitanović, J. Myrheim, Universality for period n-tuplings in complex mappings, Phys. Lett. A 94 (8) (1983) 329-333.

[21] R. Gilmore, J. W. L. McCallum, Structure in the bifurcation diagram of the Duffing oscillator, Phys. Rev. E 51 (1995) 935-956.

[22] F. G. Blake, The onset of cavitation in liquids, Tech. Rep. 12, Acoust. Res. Lab., Harvard Univ. (1949).

[23] P. L. Marston, D. B. Greene, Stable microscopic bubbles in helium i and evaporation-condensation resonance, J. Acoust. Soc. Am. 64 (1) (1978) 319-321.

[24] N. A. Gumerov, Dynamics of vapor bubbles with nonequilibrium phase transitions in isotropic acoustic fields, Phys. Fluids 12 (1) (2000) 71-88.

[25] Y. Hao, A. Prosperetti, The dynamics of vapor bubbles in acoustic pressure fields, Phys. Fluids 11 (8) (1999) 2008-2019.

[26] Z. C. Feng, L. G. Leal, Nonlinear bubble dynamics, Annu. Rev. Fluid. Mech. 29 (1) (1997) 201-243.

[27] H. C. Chang, L. H. Chen, Growth of a gas bubble in a viscous fluid, Phys. Fluids 29 (11) (1986) 3530-3536.

[28] P. Smereka, B. Birnir, S. Banerjee, Regular and chaotic bubble oscillations in periodically driven pressure fields, Phys. Fluids 30 (11) (1987) $3342-3350$. 
[29] C. E. Brennen, Cavitation and Bubble Dynamics, Oxford University Press, New York, 1995.

[30] L. Haar, J. S. Gallagher, G. S. Kell, NBS/NRC Wasserdampftafeln, Springer, Berlin, 1988.

[31] Y. C. Lai, T. Tél, Transient Chaos, Springer, New York, 2010.

[32] G. Závodszky, G. Károlyi, G. Paál, Emerging fractal patterns in a real 3D cerebral aneurysm, J. Theor. Biol. 368 (2015) 95-101.

[33] W. Lauterborn, Numerical investigation of nonlinear oscillations of gas bubbles in liquids, J. Acoust. Soc. Am. 59 (2) (1976) 283-293.

[34] U. Parlitz, V. Englisch, C. Scheffczyk, W. Lauterborn, Bifurcation structure of bubble oscillators, J. Acoust. Soc. Am. 88 (2) (1990) 1061-1077.

[35] E. A. Brujan, Bifurcation structure of bubble oscillators in polymer solutions, Acta Acust. United Acust. 95 (2) (2009) 241-246.

[36] S. Behnia, A. J. Sojahrood, W. Soltanpoor, L. Sarkhosh, Towards classification of the bifurcation structure of a spherical cavitation bubble, Ultrasonics 49 (8) (2009) 605-610.

[37] S. Behnia, A. J. Sojahrood, W. Soltanpoor, O. Jahanbakhsh, Nonlinear transitions of a spherical cavitation bubble, Chaos Solitons Fract. 41 (2) (2009) 818-828.

[38] S. Behnia, A. J. Sojahrood, W. Soltanpoor, O. Jahanbakhsh, Suppressing chaotic oscillations of a spherical cavitation bubble through applying a periodic perturbation, Ultrason. Sonochem. 16 (4) (2009) 502-511.

[39] F. Hegedüs, L. Kullmann, Basins of attraction in a harmonically excited spherical bubble model, Period. Polytech. Mech. Eng. 56 (2) (2012) 125132.

[40] A. J. Sojahrood, M. C. Kolios, Classification of the nonlinear dynamics and bifurcation structure of ultrasound contrast agents excited at higher multiples of their resonance frequency, Phys. Lett. A 376 (33) (2012) $2222-2229$. 
[41] F. Hegedüs, C. Hős, L. Kullmann, Stable period 1, 2 and 3 structures of the harmonically excited RayleighPlesset equation applying low ambient pressure, IMA J. Appl. Math. 78 (6) (2013) 1179-1195.

[42] S. Behnia, F. Mobadersani, M. Yahyavi, A. Rezavand, Chaotic behavior of gas bubble in non-Newtonian fluid: a numerical study, Nonlinear Dyn. 74 (3) (2013) 559-570.

[43] S. Behnia, H. Zahir, M. Yahyavi, A. Barzegar, F. Mobadersani, Observations on the dynamics of bubble cluster in an ultrasonic field, Nonlinear Dyn. 72 (3) (2013) 561-574.

[44] A. J. Sojahrood, O. Falou, R. Earl, R. Karshafian, M. C. Kolios, Influence of the pressure-dependent resonance frequency on the bifurcation structure and backscattered pressure of ultrasound contrast agents: a numerical investigation, Nonlinear Dyn. 80 (1-2) (2015) 889-904.

[45] R. Varga, G. Paál, Numerical investigation of the strength of collapse of a harmonically excited bubble, Chaos Solitons Fract. 76 (2015) 56-71.

[46] F. Hegedüs, K. Klapcsik, The effect of high viscosity on the collapse-like chaotic and regular periodic oscillations of a harmonically excited gas bubble (2015).

[47] U. Parlitz, W. Lauterborn, Resonances and torsion numbers of driven dissipative nonlinear oscillators, Z. Naturforsch. A 41 (4) (1986) 605614.

[48] U. Parlitz, Common dynamical features of periodically driven strictly dissipative oscillators, Int. J. Bifurcat. Chaos 3 (3) (1993) 703-715.

[49] S. Y. Kim, Bifurcation structure of the double-well Duffing oscillator, Int. J. Mod. Phys. B 14 (17) (2000) 1801-1813.

[50] U. Parlitz, W. Lauterborn, Period-doubling cascades and devil's staircases of the driven van der Pol oscillator, Phys. Rev. A 36 (3) (1987) 1428-1434.

[51] T. Kurz, W. Lauterborn, Bifurcation structure of the Toda oscillator, Phys. Rev. A 37 (1988) 1029-1031. 
[52] U. Parlitz, C. Scheffczyk, T. Kurz, W. Lauterborn, On modeling driven oscillators by maps, Int. J. Bifurcat. Chaos 1 (1) (1991) 261-264.

[53] W. Lauterborn, U. Parlitz, Methods of chaos physics and their application to acoustics, J. Acoust. Soc. Am. 84 (6) (1988) 1975-1993.

[54] E. J. Doedel, B. E. Oldeman, A. R. Champneys, F. Dercole, T. F. Fairgrieve, Y. A. Kuznetsov, R. Paffenroth, B. Sandstede, X. Wang, C. Zhang, AUTO-07P: continuation and bifurcation software for ordinary differential equations, Concordia University, Montreal, Canada (2012).

[55] C. de Boor, B. Swartz, Collocation at Gaussian points, SIAM J. Numer. Anal. 10 (4) (1973) pp. 582-606.

[56] R. D. Russell, J. Christiansen, Adaptive mesh selection strategies for solving boundary value problems, SIAM J. Numer. Anal. 15 (1) (1978) 59-80.

[57] M. M. Fyrillas, A. J. Szeri, Dissolution or growth of soluble spherical oscillating bubbles, J. Fluid Mech. 277 (1994) 381-407.

[58] W. Lauterborn, T. Kurz, Physics of bubble oscillations, Rep. Prog. Phys. 73 (10) (2010) 106501.

[59] C. Hös, A. R. Champneys, L. Kullmann, Bifurcation analysis of surge and rotating stall in the mooregreitzer compression system, IMA J. Appl. Math. 68 (2) (2003) 205-228.

[60] C. Hős, A. R. Champneys, Grazing bifurcations and chatter in a pressure relief valve model, Physica D 241 (22) (2012) 2068-2076.

[61] C. J. Hős, A. R. Champneys, K. Paul, M. McNeely, Dynamic behaviour of direct spring loaded pressure relief valves in gas service: II reduced order modelling, J. Loss Prevent. Proc. 36 (2015) 1-12.

[62] T. Uezu, Topology in dynamical systems, Phys. Lett. A 93 (4) (1983) 161-166.

[63] P. Beiersdorfer, J. M. Wersinger, Y. Treve, Topology of the invariant manifolds of period-doubling attractors for some forced nonlinear oscillators, Phys. Lett. A 96 (6) (1983) 269-272. 
[64] P. Beiersdorfer, Universality of the topology of period doubling dynamical systems, Phys. Lett. A 100 (8) (1984) 379-382.

[65] V. Englisch, W. Lauterborn, The winding-number limit of perioddoubling cascades derived as Farey-fraction, Int. J. Bifurcat. Chaos 4 (4) (1994) 999-1002.

[66] B. D. Storey, A. J. Szeri, Water vapour, sonoluminescence and sonochemistry, Proc. R. Soc. Lond. A 456 (1999) (2000) 1685-1709.

[67] A. V. Prabhu, P. R. Gogate, A. B. Pandit, Optimization of multiplefrequency sonochemical reactors, Chem. Eng. Sci. 59 (2223) (2004) 49914998.

[68] S. Koch, R. Court, W. Garen, W. Neu, R. Reuter, Detection of manganese in solution in cavitation bubbles using laser induced breakdown spectroscopy, Spectrochim, Acta B 60 (78) (2005) 1230-1235.

[69] P. Kanthale, M. Ashokkumar, F. Grieser, Sonoluminescence, sonochemistry $\left(\mathrm{H}_{2} \mathrm{O}_{2}\right.$ yield $)$ and bubble dynamics: Frequency and power effects, Ultrason. Sonochem. 15 (2) (2008) 143-150.

[70] K. Yasui, T. Tuziuti, J. Lee, T. Kozuka, A. Towata, Y. Iida, The range of ambient radius for an active bubble in sonoluminescence and sonochemical reactions, J. Chem. Phys. 128 (18) (2008) 184705.

[71] S. I. Nikitenko, L. Venault, R. Pflieger, T. Chave, I. Bisel, P. Moisy, Potential applications of sonochemistry in spent nuclear fuel reprocessing: A short review, Ultrason. Sonochem. 17 (6) (2010) 1033-1040.

[72] S. Khanna, S. Chakma, V. S. Moholkar, Phase diagrams for dual frequency sonic processors using organic liquid medium, Chem. Eng. Sci. 100 (2013) 137-144.

[73] M. Rahimi, S. Safari, M. Faryadi, N. Moradi, Experimental investigation on proper use of dual high-low frequency ultrasound wavesAdvantage and disadvantage, Chem. Eng. Process. 78 (2014) 17-26.

[74] L. Stricker, D. Lohse, Radical production inside an acoustically driven microbubble, Ultrason. Sonochem. 21 (1) (2014) 336-345. 
[75] T. J. Mason, Some neglected or rejected paths in sonochemistry A very personal view, Ultrason. Sonochem.

[76] S. Merouani, O. Hamdaoui, Y. Rezgui, M. Guemini, Sensitivity of free radicals production in acoustically driven bubble to the ultrasonic frequency and nature of dissolved gases, Ultrason. Sonochem. 22 (2015) $41-50$.

[77] R. Mettin, C. Cairós, A. Troia, Sonochemistry and bubble dynamics, Ultrason. Sonochem. 25 (2015) 24-30.

[78] D. Knorr, M. Zenker, V. Heinz, D.-U. Lee, Applications and potential of ultrasonics in food processing, Trends Food Sci. Tech. 15 (5) (2004) 261-266.

[79] R. Seshadri, J. Weiss, G. J. Hulbert, J. Mount, Ultrasonic processing influences rheological and optical properties of high-methoxyl pectin dispersions, Food Hydrocolloids 17 (2) (2003) 191-197.

[80] Y. Iida, T. Tuziuti, K. Yasui, A. Towata, T. Kozuka, Control of viscosity in starch and polysaccharide solutions with ultrasound after gelatinization, Innov. Food Sci. Emerg. 9 (2) (2008) 140-146.

[81] J. P. Canselier, H. Delmas, A. M. Wilhelm, B. Abismaïl, Ultrasound emulsification-An overview, J. Dispersion Sci. Technol. 23 (1-3) (2002) 333-349.

[82] Continuous contact- and contamination-free ultrasonic emulsification-a useful tool for pharmaceutical development and production, Ultrason. Sonochem. 13 (1) (2006) 76-85.

[83] C. H. Chaussy, W. Brendel, E. Schmiedt, Extracorporeally induced destruction of kidney stones by shock waves, Lancet 316 (8207) (1980) $1265-1268$.

[84] Z. Xu, A. Ludomirsky, L. Y. Eun, T. L. Hall, B. C. Tran, J. B. Fowlkes, C. A. Cain, Controlled ultrasound tissue erosion, IEEE Trans. Ultrason. Ferroelectr. Freq. Control 51 (6) (2004) 726-736.

[85] S. Mitragotri, Healing sound: the use of ultrasound in drug delivery and other therapeutic applications, Nat. Rev. Drug. Discov. 4 (2005) 255-260. 
[86] J. E. Kennedy, G. R. t. Haar, D. Cranston, High intensity focused ultrasound: surgery of the future?, Brit. J. Radiol. 76 (909) (2003) 590-599. 